TTP05-06

$\mathrm{SFB} / \mathrm{CPP}-05-14$

hep-ph/0505041

Mai 2005

\title{
Precise numerical evaluation of the two loop sunrise graph Master Integrals in the equal mass case.
}

\author{
S. Pozzorini and E. Remiddi* \\ Institut für Theoretische Teilchenphysik, Universität Karlsruhe, D-76128 Karlsruhe, Germany
}

\begin{abstract}
We present a double precision routine in Fortran for the precise and fast numerical evaluation of the two Master Integrals (MIs) of the equal mass two-loop sunrise graph for arbitrary momentum transfer in $d=2$ and $d=4$ dimensions. The routine implements the accelerated power series expansions obtained by solving the corresponding differential equations for the MIs at their singular points. With a maximum of 22 terms for the worst case expansion a relative precision of better than a part in $10^{15}$ is achieved for arbitrary real values of the momentum transfer.
\end{abstract}

\footnotetext{
*Supported by Alexander-von-Humboldt Stiftung permanent address: Dipartimento di Fisica, Università di Bologna, I-40126 Bologna, Italy e-mail: ettore.remiddi@bo.infn.it
} 


\section{PROGRAM SUMMARY}

Title of program: sunem

Version: 1.0

Release: 1

Catalogue identifier:

Program obtainable from: http://www-ttp.physik.uni-karlsruhe.de/Progdata/

Computers: all

Operating system: all

Program language: FORTRAN77

Memory required to execute: Size: $1532 \mathrm{k}$

No. of bits in a word: up to 32

No. of processors used: 1

No. of bytes in distributed program: 34589

Distribution format: ASCII

Other programs called: none

External files needed: none

Keywords: multi-loop Feynman integrals, differential equations

Nature of the physical problem: numerical evaluation of the two Master Integrals of the equal mass two-loop sunrise Feynman graph for arbitrary momentum transfer in $d=2$ and $d=4$ dimensions.

Method of solution: accelerated power series expansions obtained by solving the differential equations for the MIs at their singular points. With a maximum of 22 terms for the worse case expansion a relative precision of better than a part in $10^{15}$ is achieved for arbitrary real values of the momentum transfer.

Restrictions on complexity of the problem: limited to real momentum transfer and equal internal masses.

Typical running time: approximately $1 \mu$ s to evaluate the four Master integrals for a fixed momentum transfer value on a Pentium IV/3 GHz Linux PC. 


\section{LONG WRITE-UP}

\section{Introduction.}

The continuous progress in the evaluation of radiative correections to various elementary particle processes, driven by the increasing number of current and future precise experimental results, is faced with the problem of the fast and precise numerical evaluation of those radiative corrections that cannot be expressed in terms of known analytic functions for which numerical routines are already available.

In this paper we discuss a FORTRAN routine for the precise and fast numerical evaluation of the two Master Integrals (MIs) associated with the two-loop selfmass sunrise graph for equal masses, based on the results of [1], where their analytic properties were established by studying the linear system of first order inhomogeneous differential equations satisfied by the MIs themselves.

As a first obvious step, the linear system can be rewritten as a second order (inhomogeneous) linear differential equation for one of the MIs (from now on referred to as the main MI, or simply the MI), while the other MI can be expressed in terms of the first and its derivative. The accurate investigation of the second order equation shows that the immaginary part of the MI (which satisfies the associated homogeneous equation) is an ellyptic function of complicated argument (a fact known however since a long time), while the real part is likely to belong to a new family of analytic functions, which could not be traced back to the usual Nielsen's polylogarithms nor to their generalizations [2].

Nevertheless, the differential equation can be used to obtain a convenient integral representation of the solution and, among other things, the explicit limiting behaviour of the MI under investigation at zero momentum transfer, at the pseudothreshold, at the threshold and at asymptotic momenta. Given those limiting values as starting points, the differential equation can then be used to generate the coefficients of the relevant expansions up to any required order.

In this paper we show that those expansions can be used for writing a (double precision) FORTRAN routine for the fast and precise numerical evaluation of both the MIs, within the $d$ continuous regularization scheme, in $d=2$ and $d=4$ dimensions. According to [1, it is indeed sufficient to work out the relevant expansions only for the main MI in $d=2$ dimensions by direct iterative solution of the differential equation; the other MI in $d=2$ dimensions, as well as both MIs in $d=4$ dimensions, are then expressed in terms of the first MI in $d=2$ dimensions and its derivatives (needless to say, differentiation becomes a simple algebraic operation when dealing with expansions).

In the next 3 Sections we discuss the relevant expansions of the first MI in $d=2$ dimensions for physical momentum around zero, at threshold and at infinity. In Section 6 we describe how to use the results of the previous Section for implementing the FORTRAN routine for the evaluation of the two MIs at $d=2$ and $d=4$ dimensions. In order to reach a relative precision of better than $1 \times 10^{-15}$ (which is essentialy the limit of double precision FORTRAN) for any value of the squared momentun transfer $s$ in the whole real axis, $-\infty<s<+\infty$, it is sufficient to keep a maximum of 22 terms in the various expansions.

\section{Definition and notation.}

The two MIs associated with the (equal mass) 2-loop sunrise graph depicted in Fig. 10are defined, in the $d$-continuous regularization scheme, as

$$
\begin{aligned}
S(d, z) & =\int \frac{\mathcal{D}^{d} k_{1} \mathcal{D}^{d} k_{2}}{\left(k_{1}^{2}+1\right)\left(k_{2}^{2}+1\right)\left[\left(p-k_{1}-k_{2}\right)^{2}+1\right]}, \\
S_{1}(d, z) & =\int \frac{\mathcal{D}^{d} k_{1} \mathcal{D}^{d} k_{2}}{\left(k_{1}^{2}+1\right)^{2}\left(k_{2}^{2}+1\right)\left[\left(p-k_{1}-k_{2}\right)^{2}+1\right]},
\end{aligned}
$$




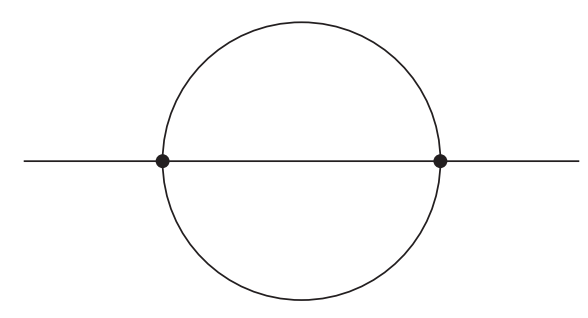

Figure 1: The 2-loop sunrise graph.

where

$$
\mathcal{D}^{d} k=\frac{1}{\Gamma\left(3-\frac{d}{2}\right)} \frac{\mathrm{d}^{d} k}{4 \pi^{\frac{d}{2}}},
$$

$p$ is the external momentum, $p^{2}=z=-s$ (when $p$ is Euclidean or spacelike, $z$ is positive and $s$ is negative). One has, for arbitrary $d$

$$
S_{1}(d, z)=\frac{1}{3}\left[-(d-3)+z \frac{d}{d z}\right] S(d, z) .
$$

At $d=2 S(d, z)$ and $S_{1}(d, z)$ are both finite,

$$
\begin{aligned}
S(d, z) & =S(2, z)+\mathcal{O}(d-2), \\
S_{1}(d, z) & =S_{1}(2, z)+\mathcal{O}(d-2),
\end{aligned}
$$

and from now on we will simply consider $S(2, z), S_{1}(2, z)$. $S(2, z)$ satisfies the second order inhomogenous equation

$$
\left\{\frac{d^{2}}{d z^{2}}+\left[\frac{1}{z}+\frac{1}{z+1}+\frac{1}{z+9}\right] \frac{d}{d z}+\left[\frac{1}{3 z}-\frac{1}{4(z+1)}-\frac{1}{12(z+9)}\right]\right\} S(2, z)=\frac{3}{8 z(z+1)(z+9)},
$$

which will be used to derive all the required expansions.

Around $d=4 S(d, z)$ develops a double pole in $(d-4)$, so that it can be written as

$$
S(d, z)=\frac{1}{(d-4)^{2}} S^{(-2)}(4, z)+\frac{1}{d-4} S^{(-1)}(4, z)+S^{(0)}(4, z)+\mathcal{O}(d-4) .
$$

From [1] one has

$$
\begin{aligned}
S^{(-2)}(4, z)= & -\frac{3}{8} \\
S^{(-1)}(4, z)= & \frac{1}{32}(z+18) \\
S^{(0)}(4, z)= & \frac{1}{12}(z+1)(z+9)\left(1+(z-3) \frac{d}{d z}\right) S(2, z) \\
& -\frac{1}{128}(72+13 z) .
\end{aligned}
$$

From Eq.(3), recalling that $S_{1}(2, z)$ is finite while $S_{1}(d, z)$ can be expanded around $d=4$ as $S(d, z)$ Eq.(6), we find

$$
S_{1}(2, z)=\frac{1}{3}\left[1+z \frac{d}{d z}\right] S(2, z)
$$




$$
\begin{aligned}
S_{1}^{(-2)}(4, z) & =\frac{1}{8} \\
S_{1}^{(-1)}(4, z) & =-\frac{1}{16}, \\
S_{1}^{(0)}(4, z) & =\frac{1}{3}\left[-1+z \frac{d}{d z}\right] S^{(0)}(4, z)-\frac{1}{96}(z+18) .
\end{aligned}
$$

We will work out explicitly, from the differential equations, the expansions of $S(2, z)$ in the various regions; we will then use Eq.s (879) for obtaining the expansions of $S_{1}(2, z), S^{(0)}(4, z)$ and $S_{1}^{(0)}(4, z)$ in terms of the corresponding expansions of $S(2, z)$.

\section{The expansion around $z=0$.}

The point $z=0$ ( or $s=0$ ) is a singular point of the differential equation Eq. (5); the investigation carried out in 1 shows that the most general solution $G(z)$ consists of a regular part and a singular part behaving like $\ln z$ at $z=0$, so that its expansion is

$$
G(z)=\sum_{n=0}^{\infty} a_{n} z^{n}+\ln z \sum_{n=0}^{\infty} b_{n} z^{n} .
$$

Once the expansion Eq. (10) is substituted in Eq.(5), the differential equation takes the form of a system of linear algebraic equations for the two sets of coefficients $a_{n}, b_{n}$, and can be solved recursively expressing the coefficient of order $n$ in terms of the coefficients of lower order. The procedure requires two initial conditions, i.e. the two coefficients $a_{0}$ and $b_{0}$.

As discussed in [1] in the case of $S(2, z)$ the two initial conditions are

$$
\begin{aligned}
a_{0} & =\frac{\sqrt{3}}{12} \mathrm{Cl}_{2}\left(\frac{\pi}{3}\right)=0.146494 \ldots, \\
b_{0} & =0,
\end{aligned}
$$

where $\mathrm{Cl}_{2}\left(\frac{\pi}{3}\right)$ is the Clausen function of argument $\pi / 3$, while the vanishing of $b_{0}$ is due to the regularity of $S(2, z)$ at $z=0$.

Therefore, the expansion for $S(2, z)$ reads simply

$$
S(2, z)=\sum_{n=0}^{\infty} a_{n} z^{n}
$$

and on account of the explicit value of $a_{0}$ provided by Eq.(11) the differential equation then gives for the higher order coefficients

$$
\begin{aligned}
& a_{1}=-\frac{\sqrt{3}}{36} \mathrm{Cl}_{2}\left(\frac{\pi}{3}\right)+\frac{1}{24}=-0.00716473 \ldots \\
& a_{2}=\sqrt{3} \frac{5}{324} \mathrm{Cl}_{2}\left(\frac{\pi}{3}\right)-\frac{23}{864}=0.000508185 \ldots \\
& a_{3}=-\sqrt{3} \frac{31}{2916} \mathrm{Cl}_{2}\left(\frac{\pi}{3}\right)+\frac{145}{7776}=-0.0000414414 \ldots
\end{aligned}
$$

and so on. The rational fractions (such as $1 / 24$ in $a_{1}$ ) are the expansion of the solution of the complete inhomogenous equation Eq. (15) with initial condition $S(2,0)=0$, whereas the irrational part with $\mathrm{Cl}_{2}\left(\frac{\pi}{3}\right)$, which is proportional to the regular solution of the associated homogenous equation, is fixed by the initial conditions.

The nearest singular point of the differential equation Eq.(5) is at $z=-1(s=1)$, so one would expect a convergence radius 1 for the expansion Eq. (12); but the nearest singularity of $S(2, z)$ 
is at the physical threshold $s=9$ or $z=-9$, so that $S(2, z)$ is analytic at the pseudothreshold $z=-1$; that is achieved by suitable cancellations among the two (rational and irrational) terms which appear in the explicit expressions of the coefficients $a_{n}$, as already shown also by the first coefficients Eq. 131).

The next singular point of the differential equation is at $z=-9$ (or $s=9$ ), which is also a non analyticity point for $S(2, z)$, as commented above; the expansion Eq.(12) has therefore convergence radius 9 .

The knowledge of the position of the nearest singularity allows to greatly speed up the convergence of the series, by introducing the "Bernoulli" variable (first proposed in 3 for the evaluation of Euler's dilogarithm, and repeatedly used in 4 for the evaluation of Harmonic Polylogarithms), which is given in this case by

$$
t=\ln \left(1+\frac{z}{9}\right)
$$

One has

$$
z=9\left(\mathrm{e}^{t}-1\right)=9\left(t+\frac{t^{2}}{2}+\frac{t^{3}}{6}+\ldots\right)
$$

replacing $z$ in Eq.(12) by its expansion in $t$, one obtains a new expansion in $t$ which we write as

$$
S(2, z)=\sum_{n=0}^{\infty} \alpha_{n} t^{n} .
$$

The explicit knowledge of $N$ terms of the expansion Eq.(12) detrmines the first $N$ terms of Eq.(15), which for a same number of expansion terms and small $t$ (see Section 6 for more details) approximates $S(2, z)$ much better than the original expansion in $z$. A straightforward calculation gives for the first coefficients

$$
\begin{aligned}
& \alpha_{0}=a_{0}=\frac{\sqrt{3}}{12} \mathrm{Cl}_{2}\left(\frac{\pi}{3}\right)=0.146494 \ldots \\
& \alpha_{1}=\frac{3}{8}-\frac{\sqrt{3}}{4} \mathrm{Cl}_{2}\left(\frac{\pi}{3}\right)=-0.0644826 \ldots \\
& \alpha_{2}=\sqrt{3} \frac{9}{8} \mathrm{Cl}_{2}\left(\frac{\pi}{3}\right)-\frac{63}{32}=0.00892173 \ldots \\
& \alpha_{3}=-\sqrt{3} \frac{157}{24} \mathrm{Cl}_{2}\left(\frac{\pi}{3}\right)+\frac{23}{2}=0.000205110 \ldots,
\end{aligned}
$$

and so on. Note the strong cancellation between the two terms in each coefficient of the expansion; the cancellation, already present in Eq.(13) as remarked above, is even bigger in Eq. (16), and grows quickly with the order $n$ of the coefficient $\alpha_{n}$. Keeping the control of cancellations in the numerical evaluation of the coefficients would be in principle a delicate task, but that is no longer problem when using the arbitrary precision features of Mathematica [5].

\section{The expansion around $z=-9$.}

As already remarked, the point $z=-9(s=9)$ is the nearest singular point of $S(2, z)$. For $z \rightarrow-9$, the general solution consists of a regular part and a singular part behaving like $\ln (z+9)$, so that $S(2, z)$ admits an expansion of the general form

$$
S(2, z)=\sum_{n=0}^{\infty} a_{n}(z+9)^{n}+\ln (z+9) \sum_{n=0}^{\infty} b_{n}(z+9)^{n},
$$

where the coefficients, called again $a_{n}, b_{n}$ as in Eq.(10), are of course different from the coefficients of the expansion at $z=0$. For $z>-9$, i.e. $s<9$ or $s$ below threshold, both terms of Eq. (17) are 
real, while for $z<-9$, or $s>9$, above threshold, $\ln (z+9)$ develops an immaginary part, which with the usual $s+i \epsilon$ prescription is $-i \pi$,

$$
\ln (z+9)=\ln |z+9|-i \pi \theta(-z-9) .
$$

From 1] we have the initial conditions

$$
\begin{aligned}
& a_{0}=-\frac{\sqrt{3}}{48} \pi, \\
& b_{0}=\frac{\sqrt{3}}{48}\left[\pi \ln (72)-5 \mathrm{Cl}_{2}\left(\frac{\pi}{3}\right)\right] .
\end{aligned}
$$

From the differential equation we found for the first coefficients

$$
\begin{aligned}
a_{1} & =a_{0} \frac{1}{12}+b_{0} \frac{5}{72}+\frac{1}{192}, \\
b_{1} & =b_{0} \frac{1}{12}, \\
a_{2} & =a_{0} \frac{7}{864}+b_{0} \frac{97}{10368}+\frac{5}{6912}, \\
b_{2} & =b_{0} \frac{7}{864},
\end{aligned}
$$

and so on for the higher order coefficients. As in the previous section, the terms independent of the initial conditions $a_{0}, b_{0}$ (such as the fraction 1/192 in $a_{1}$ ) are due to the inhomogenous term in the differential equation Eq.(5).

The nearest singular point for the two components of the expansion Eq.(17) is at $z=-1$, so that the radius of convergence of the two expansions is 8 , i.e. the expansions converge in the range $-17<z<-1$ or $1<s<17$. That might look surprising, as $S(2, z)$ is analytic at $z=-1$; but the two terms in the expansion Eq.(17) cannot combine to a single expansion converging in a wider range, given the presence of the $\ln (z+9)$ in front of the second term.

In this case the "Bernoulli" variable is therefore

$$
t=-\ln \left(1-\frac{z+9}{8}\right),
$$

and the expansion of $S(2, z)$ in terms of $t$ reads

$$
S(2, z)=\sum_{n=0}^{\infty} \alpha_{n} t^{n}+\ln (z+9) \sum_{n=0}^{\infty} \beta_{n} t^{n} .
$$

The explicit calculation gives

$$
\begin{aligned}
\alpha_{0} & =a_{0}=0.301695 \ldots, \\
\beta_{0} & =b_{0}=-0.113362 \ldots, \\
\alpha_{1} & =\frac{2}{3} a_{0}+\frac{5}{9} b_{0}+\frac{1}{24}=0.179817 \ldots, \\
\beta_{1} & =\frac{2}{3} b_{0}=-0.0755749 \ldots, \\
\alpha_{2} & =\frac{5}{27} a_{0}+\frac{26}{81} b_{0}+\frac{11}{432}=0.0449445 \ldots, \\
\beta_{2} & =\frac{5}{27} b_{0}=-0.0209930 \ldots,
\end{aligned}
$$

with the by now usual strong cancellations within the various terms of each coefficient. 


\section{The expansion at infinity.}

The differential equation has also $z=\infty$ as singular point; for $z \rightarrow \infty$ the expected behaviour for $S(2, z)$ is

$$
S(2, z)=\frac{1}{z}\left(\sum_{n=0}^{\infty} a_{n} \frac{1}{z^{n}}+\ln z \sum_{n=0}^{\infty} b_{n} \frac{1}{z^{n}}+\ln ^{2} z \sum_{n=0}^{\infty} c_{n} \frac{1}{z^{n}}\right) .
$$

For $z>0$ (i.e. $s$ spacelike) the previous expression is real; in the timelike region, we have to replace $z$ by $z-i \epsilon$; that gives

$$
\begin{aligned}
\ln z & =\ln |z|-i \pi \theta(-z), \\
\ln ^{2} z & =\ln ^{2}|z|-\theta(-z)\left(2 i \pi \ln |z|+\pi^{2}\right),
\end{aligned}
$$

from which one can obtain separately the real and immaginary parts of $S(2, z)$.

From [1] the initial conditions are

$$
\begin{aligned}
a_{0} & =0, \\
b_{0} & =0, \\
c_{0} & =\frac{3}{16} .
\end{aligned}
$$

It is to be noted that the presence of the term $3 / 16 \ln ^{2} z$ is anyhow required by the inhomogenous term in the differential equation, so that strictly speaking the initial conditions are simply $a_{0}=$ $b_{0}=0$. The differential equation then gives for the first coefficients

$$
\begin{aligned}
& a_{1}=\frac{3}{8}, \\
& b_{1}=\frac{3}{2}, \\
& c_{1}=-\frac{9}{16}, \\
& a_{2}=-\frac{3}{32}, \\
& b_{2}=-\frac{39}{4}, \\
& c_{2}=\frac{45}{16},
\end{aligned}
$$

and so on for the higher orders.

As the nearest singularity is at $z=-9$, the "Bernoulli" variable is

$$
t=\ln \left(1+\frac{9}{z}\right),
$$

and we write the expansion of $S(2, z)$ in terms of $t$ as

$$
S(2, z)=\left(\sum_{n=0}^{\infty} \alpha_{n} t^{n}+\ln z \sum_{n=0}^{\infty} \beta_{n} t^{n}+\ln ^{2} z \sum_{n=0}^{\infty} \gamma_{n} t^{n}\right),
$$

so that the first coefficients are

$$
\begin{aligned}
& \alpha_{0}=\beta_{0}=\gamma_{0}=0, \\
& \alpha_{1}=a_{0}=0, \\
& \beta_{1}=b_{0}=0,
\end{aligned}
$$




$$
\begin{aligned}
& \gamma_{1}=\frac{1}{48}, \\
& \alpha_{2}=\frac{1}{216}, \\
& \beta_{2}=\frac{1}{54}, \\
& \gamma_{2}=\frac{1}{288}, \\
& \alpha_{3}=\frac{35}{776}, \\
& \beta_{3}=\frac{5}{972}, \\
& \gamma_{3}=\frac{1}{2592},
\end{aligned}
$$

etc.

\section{The FORTRAN numerical routine.}

As a first step, we obtained the analytic expression of the coefficients of all the needed expansions for $S(2, z)$ up to the required order (see below). From those we obtained the corresponding expansions for the other three quantities, $S_{1}(2, z), S^{(0)}(4, z)$ and $S_{1}^{(0)}(4, z)$ by means of the formulae Eq.s 879), which involve only elementary algebra and differentiation. When dealing with power series expansions, differentiating is trivial, even if one term is lost at each differentiation; but that is easily compensated by allowing for a few extra terms in the initial expansion.

We then evaluated the numerical value of all the coefficients so obtained. The analytic and numerical evaluations were carried out by Mathematica, in arbitrary precision arithmetics mode for keeping rounding errors under check. The numerical values of the coefficients were transferred to the FORTRAN code as constants with 18 digits; that exceeds of the course the double precision of FORTRAN but eliminates rounding problems in the least significant digit.

In the range $-\infty<z<-17.45$, or $17.45<s<+\infty$ (timelike region above threshold, all MIs complex), we evaluate the 4 functions by means of truncated expansions of the form

$$
\sum_{n=0}^{N} \alpha_{n} t^{n}+\ln z \sum_{n=0}^{N} \beta_{n} t^{n}+\ln ^{2} z \sum_{n=0}^{N} \gamma_{n} t^{n},
$$

where $t$ is defined by Eq. (28) and varies therefore in the range $-0.725173 \ldots<t<0$, the complex value of $\ln z$ is specified in Eq.(25) and the values of the coefficients $\alpha_{n}, \beta_{n}, \gamma_{n}$ depend of course on the MIs. We obtain the required double precision (relative error less than $1 \times 10^{-15}$ ) for all the 4 functions with $N=22$. Those numerical checks, beyond the possibilities offered by double precision FORTRAN, were carried out by using Mathematica in arbitrary precision mode and including a growing number of terms in the expansion.

In the range $-17.45 \leq z<-5.15$, or $5.15<s \leq 17.45$ (timelike region across the threshold $s=9$ ) we use for the 4 functions truncated expansions of the form

$$
\frac{R}{z+9}+\sum_{n=0}^{N} \alpha_{n} t^{n}+\ln (z+9) \sum_{n=0}^{N} \beta_{n} t^{n},
$$

where $R=-\sqrt{3} \pi / 48$ for $S_{1}(2, z)$ and vanishes in all the other cases, $t$, defined in Eq.(21), varies in the range $-0.720884 \ldots \leq t<0.656333 \ldots$ and the value of $\ln (z+9)$ is given in Eq. (18). The required precision (better than $1 \times 10^{-15}$ ) is obtained with $N=21$. 
In the range $-5.15 \leq z<11$, or $-11<s \leq 5.15$, we use truncated expansions of the form

$$
\sum_{n=0}^{N} \alpha_{n} t^{n},
$$

where $t$, defined in Eq.(14), varies in the range $-0.849151 \leq t<0.798508$, and the required precision is obtained with $N=18$. Note that the expansion in $t$ is valid, and still quickly convergent, for corresponding values of $z$ also in the region $9<z<11$, which lies outside the region of convergence of the expansion in $z$ from which the expansion in $t$ was derived!

Finally, in the range $11 \leq z<+\infty$, or $-\infty<s \leq-11$ (spacelike region), we use again the expansion Eq.(31), with $t$ varying in the range $0<t \leq 0.597837 \ldots$; in this case $\ln z$ is real, and the aimed precision is obtained with $N=20$.

The numerical values of the 4 functions, evaluated according to the previous formulas, are returned by calling the FORTRAN routine

SUBROUTINE SUNRISE (S, SR2, SI2, S1R2, S1I2, SR4 , SI4 , S1R4, S1I4) ,

where all the variables are real DOUBLE PRECISION variables, $\mathrm{S}$ is the momentum transfer (S, equal to $-z$ of previous sections, is positive when timelike, with physical threshold at 9), SR2, SI2 are the real and immaginary parts of $S(2, z)$ as defined in Eq.(1) for $d=2$ and $z=-\mathrm{S}, \mathrm{S} 1 \mathrm{R} 2, \mathrm{~S} 1 \mathrm{I} 2$ the real and immaginary parts of $S_{1}(2, z)$ as defined also in Eq.(11), SR4, SI4 the real and immaginary parts of $S^{(0)}(4, z)$ as defined by Eq.S(16), and S1R4, S1I4 finally the real and immaginary parts of $S_{1}^{(0)}(4, z)$. S can vary in the whole real axis, $-\infty<\mathrm{S}<+\infty$; the immaginary parts, which appear when $\mathbf{S}$ is above threshold $(\mathrm{S}>9)$, are evaluated according to the standard $\mathbf{S}+i \epsilon$ prescription.

The calculation is carried out with relative precision better than $1 \times 10^{-15}$ (which is essentialy the double precision limit of FORTRAN), except of course when the corresponding quantities approach zero. $S(2, z)$ and $S_{1}(2, z)$ are positive definite and tend to zero for $|z| \rightarrow \infty$, while $S^{(0)}(4, z)$ and $S_{1}^{(0)}(4, z)$ have a zero around $z=12.5910 \ldots$ and $z=-3.5599 \ldots$, respectively.

Internally, the routine SUNRISE calls, depending on the actual value of $\mathrm{S}$, one of the three subroutines SUNRISE0, SUNRISE9 and SUNRISE00. Those routines implement the various expansions as discussed in the previous sections. For ease of check and implementation, the routines contain more coefficients than actually used, each coefficient being an entry in a DATA statement written as a constant with 18 decimal digits (as already remarked above, that exceeds of course the double precision of FORTRAN but eliminates rounding problems). The arguments of the three routines consist of the same arguments of SUNRISE and an extra integer argument, specifying how many coefficients of the expansions are to be considered in the calculation. The three internal routines can in principle be called independently, for studying the contributions of the various terms of the expansions etc., as well as for additional checks.

As a last remark, we succeeded in covering the whole real axis in $z$ with just three different expansions and a maximum of 22 terms at worst; that is fully satisfactory, and from the practical point of view there is no need of further improvements. But let us observe that, in principle, the calculation could be further speeded up by using additional auxiliary expansions. For instance, one could consider another expansion point, say for instance at $z=-18$ (or $s=18$ ); as that is a regular point in which the function is analytic, one can write as

$$
A+B(z+18)+\mathcal{O}\left((z+18)^{2}\right)
$$

the limiting behaviour of the function to be evaluated, parametrizing it in terms of two initial constants $A, B$, still to be determined. By using the differential equation, it is immediate to generate as many coefficients as desired of the expansion in powers of $(z+18)$. The two constants can then be obtained by matching numerically the new expansion with the expansion around $z=-9$ at, say, $z=-13$, and with the expansion at $z=-\infty$ at, say, $z=24$. In so doing, one has to use every involved expansion on a smaller range, where the desired precision can be achieved with a smaller number of terms. 
By the same token, one could consider the expansion around $z=-9$ Eq. (17) and keep the initial conditions $a_{0}, b_{0}$, whose exact value is given by Eq.(19), as still unknown constants, use the differential equation to work out the desired coefficients of the expansion around $z=-9$, and then obtain numerically $a_{0}, b_{0}$ by imposing the smooth matching at, say, $z=-5$ with the value obtained with the expansion around $z=0$, supposedly known. One could then continue to recover in that manner the numerical value of the initial constants of the expansion at infinity, supposedly unknown as well. Note that in this procedure one can insert as many auxiliary expansions as considered useful; but one cannot skip the expansions at the singular points of the function, as those points cannot be included, by the very definition of singular points, in the convergence radius of any expansion around nearby points. The differential equation, again, gives the most general behaviour of the function at any point, to be used for obtaining the expansion around that point.

\section{$7 \quad$ Example}

The following simple program illustrates how to evaluate the real and imaginary parts of the Master integrals $S(2, z), S_{1}(2, z), S^{(0)}(4, z)$ and $S_{1}^{(0)}(4, z)$ for a given value of the momentum transfer $s=-z$ :

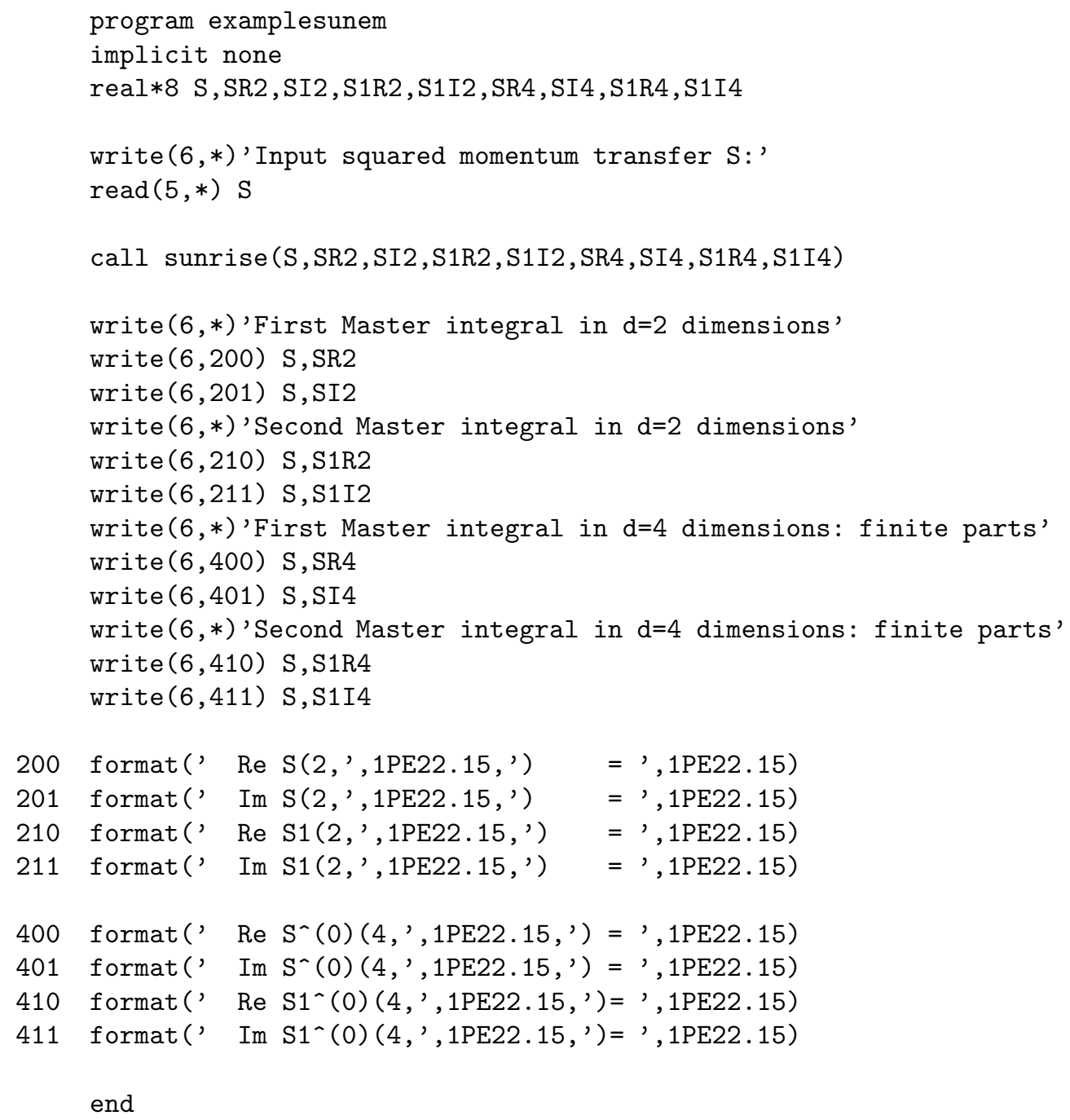




\section{Acknowledgements}

This work was supported in part by the Deutsche Forschungsgemeinschaft in the SFB/TR 09-03.

\section{References}

[1] S. Laporta and E. Remiddi, Nucl. Phys. B 704 (2005) 349 hep-ph/0406160.

[2] E. Remiddi and J. A. M. Vermaseren, Int. J. Mod. Phys. A 15 (2000) 725 hep-ph/9905237.

[3] G. 't Hooft and M. J. G. Veltman, Nucl. Phys. B 153 (1979) 365.

[4] T. Gehrmann and E. Remiddi, Comput. Phys. Commun. 141 (2001) 296 hep-ph/0107173.

[5] S. Wolfram, Mathematica Version 4.2. 